\title{
Improving quality of life in self-reported gastrointestinal disorders: An open trial of a food elimination diet guided by the ImmunoBloodprint food sensitivity test ${ }^{*}$
}

\author{
Judi M. Woolger ${ }^{1}$, Johanna Lopez ${ }^{2}$, Angelica B. Melillo ${ }^{3}$, Eduard Tiozzo ${ }^{3}$, Yaima Alonso ${ }^{3}$, \\ Soyona Rafatjah ${ }^{3}$, Amine Sarabia ${ }^{3}$, Susanna M. Leonard ${ }^{3}$, Evan G. Long ${ }^{3}$, Michael Schoor ${ }^{1}$, \\ Jared Tannenbaum ${ }^{1}$, Janet Konefal ${ }^{3}$, John E. Lewis ${ }^{3}$ \\ ${ }^{1}$ Department of Medicine, University of Miami Miller School of Medicine, Miami, USA \\ ${ }^{2}$ Department of Dietetics and Nutrition, Florida International University, Miami, USA \\ ${ }^{3}$ Department of Psychiatry \& Behavioral Sciences, University of Miami Miller School of Medicine, Miami, USA \\ Email: jelewis@miami.edu
}

Received 31 May 2013; revised 30 June 2013; accepted 14 July 2013

Copyright (C) 2013 Judi M. Woolger et al. This is an open access article distributed under the Creative Commons Attribution License, which permits unrestricted use, distribution, and reproduction in any medium, provided the original work is properly cited

\begin{abstract}
Purpose: Irritable bowel syndrome (IBS) and gastrointestinal (GI) disorders are genuine health problems that have serious consequences on a patient's quality of life (QoL). IBS is a functional disorder characterized by chronically recurring symptoms, including abdominal pain or discomfort, altered stool frequency and consistency, and abdominal bloating in the absence of structural or biochemical abnormalities. Studies have indicated that symptoms and disability related to IBS and GI disorders correlate with QoL measures. Additionally, those who respond to therapy have an improvement in QoL. Therefore, we explored an open trial of a food elimination diet guided by the ImmunoBloodprint food sensitivity test and its effect on QoL in patients self-reporting an unresolved GI disorder. Methods: Eighty-four subjects aged 18 and above participated in the study. Subjects eliminated all IgG-mediated reactive foods from their diet for $\mathbf{9 0}$ days. QoL was assessed with the Irritable Bowel Syndrome-36 (IBS-36) and the Medical Outcomes Study Short Form-36 (SF-36) at baseline and 30-, 60-, and 90-day follow-up. The data were analyzed with linear mixed models. Results: Subjects had statistically significant improvements in all indicators of QoL according to the IBS-36 and SF-36 from baseline to 90day follow-up. Conclusions: Subjects with unresolved self-reported GI disorders were able to improve all indicators of QoL in response to eliminating IgG-
\end{abstract}

\footnotetext{
We have no financial disclosures to report regarding the conduct of this research.
}

reactive foods from the diet. Given the inconvenience of keeping track of foods and symptoms to eliminate intolerant foods, a food sensitivity test combined with an elimination diet may be an effective method for improving subjective markers of QoL and health.

Keywords: GI Disorders; Food Sensitivity Testing; Elimination Diet; Food Intolerance; IBS-36; SF-36

\section{INTRODUCTION}

Gastrointestinal (GI) disorders, such as irritable bowel syndrome (IBS), are usually non-life-threatening conditions. Even though they generally do not require surgery and do not result in increased mortality, these conditions can cause health problems with serious impairments on a patient's quality of life (QoL) [1]. In fact, the general health status of American and British patients with IBS is worse compared to that of the general population in each country [2]. For instance, young American adults with IBS had a significant reduction in health-related QoL, as evidenced by lower scores on the SF-36 survey compared to the US population [2]. A systematic review of IBS patients concluded that those who respond to therapy have a corresponding improvement in health-related QoL [3]. For example, GI medications, such as Alosetron and Mebeverine, significantly improve symptoms and QoL compared to placebo [4-8]. These data support the view that symptoms and disability related to GI disorders correlate with QoL, suggesting that those who respond to therapy have an improvement in QoL.

The decrease in QoL measures also equates to an eco- 
nomic burden on the workplace and healthcare system. IBS is one of the most frequently diagnosed GI disorders in primary care and gastroenterology practices [9]. It affects $3 \%-20 \%$ of the US population with women affected twice as much as men, and the peak incidence is between 15 and 34 years of age $[10,11]$. The total indirect and direct cost of IBS is estimated to be $\$ 30$ billion annually, which is higher than that for other chronic conditions such as asthma. Patients with IBS have a tendency to seek medical care for non-GI symptoms more often than individuals without IBS [9].

Currently, recommendations of the American College of Gastroenterology and the British Society of Gastroenterology for the treatment of functional GI disorders include an initial step (i.e., diagnosis) followed by three secondary steps that involve pharmacological treatment (i.e., antidepressants, antispasmodics, and other medications that affect bowel movement like anti-diarrheals), psychological evaluation, and lifestyle/dietary modifications. Dietary or medical nutrition therapy is usually an integral part of the approach to treat these conditions, since the symptoms of GI disorders are typically subsequent to food ingestion. Thus, a diet that eliminates intolerant foods is usually beneficial for the patient. However, detecting intolerant foods usually requires a patient to record food intake and any symptoms associated with food ingestion, which may be impractical and inconvenient. Therefore, a clinical test detecting intolerant foods can represent a more efficient approach.

IBS and similar GI disorders may be low-grade inflammatory bowel diseases with altered immune activation. Efforts to test for food intolerance in patients with functional GI disorders have focused on the Immunoglobulin E (IgE)-mediated food allergy response, but this immediate-type reaction seems to be rare [12-14]. Therefore, adverse reactions to food observed in patients with functional GI disorders may be due to a delayed immunological response involving Immunoglobulin G (IgG) antibodies, which are reported to be causal in several food hypersensitivities [15-18]. The ImmunoBloodprint is a novel food sensitivity test that detects the presence of IgG antibodies to specific foods.

We hypothesized that an individualized elimination diet based on the IgG-reactive foods detected by the ImmunoBloodprint would improve QoL and GI-specific symptoms in patients with self-reported GI disorders. Therefore, the purpose of this study was to evaluate changes in health-related QoL, as assessed by the Irritable Bowel Syndrome-36 (IBS-36) and the Medical Outcomes Study Short Form-36 (SF-36) surveys in patients with unresolved self-reported GI disorders following an immunologically-reactive food elimination diet based on the ImmunoBloodprint results.

\section{METHODS}

\subsection{Subjects}

Potential subjects $(\mathrm{n}=84)$ who self-reported an unresolved GI disorder lasting more than 6 months, e.g., IBS, diarrhea, constipation, gas, or bloating, were identified through referrals and advertisements at the University of Miami Miller School of Medicine between 2008 and 2010. The study was conducted with the approval of the Institutional Review Board for human subjects research, and all participants signed informed consent and HIPAA forms before commencing in the study. The sample was comprised of $18 \%$ males $(n=15)$ and $82 \%$ females $(n=$ 69) with a mean age of 45.6 years $(\mathrm{SD}=12.8 ; \mathrm{R}=19$, 68). The racial/ethnic distribution of the sample was as follows: $48.1 \%$ Hispanic $(n=37), 44.2 \%$ white, nonHispanic $(\mathrm{n}=34), 6.5 \%$ black, non-Hispanic $(\mathrm{n}=5)$, and $1.3 \%$ other groups $(\mathrm{n}=1)$.

\subsection{Study Design}

Subjects were enrolled in the study if they were: 1 ) older than 18 years of age; 2) not currently participating in another research trial for GI problems; 3) not suffering from serious medical complications that might limit their participation, such as recent heart attack, stroke, or chronic kidney disease; 4) not pregnant; and/or 5) willing to eliminate the reactive foods from their diet for 90 days based on the results of the ImmunoBloodprint test.

Each eligible subject was scheduled for a blood draw, and the blood sample was sent to Immuno Laboratories, Inc. (Ft. Lauderdale, FL) for processing and analysis. Immuno Laboratories, Inc. is accredited by the College of American Pathologists, licensed federally and in several states, and utilizes a proprietary test known as the ImmunoBloodprint. The ImmunoBloodprint test uses microtiter plates with tiny wells that hold antigens of 115 commonly eaten foods and ingredients (see Appendix 1). The blood is tested with each antigen. Laser-like light beamed on a micro plate reads precisely which foods are reactive to each participant's blood based on IgG responses to each antigen.

Subjects were provided with both the test results and an individualized dietary plan based on replacing IgGreactive foods with non-reactive foods as per the ImmunoBloodprint results, when they returned to complete the baseline assessment. A personalized rotation plan of their non-reactive foods and general information about healthy eating, food preparation, and shopping were given to all participants. No other behaviors were addressed in the instructions. Primarily, participants were advised to focus as much as possible on eliminating their reactive foods from the diet for the entire 90-day period. All participants were encouraged to contact the study team with any dietary related questions. 


\subsection{Outcomes and Assessment Schedule}

Each participant completed a basic demographics and medical history questionnaire at baseline and recorded any changes in their medications during the course of the study. Criteria used to select the assessment instruments included: 1) appropriateness for the population; 2) ease of administration and scoring; 3 ) experience administering these measures, and 4) employment of measures involving a multi-method (i.e., self-report and physical measures) approach to enhance the validity of the overall assessment.

The primary outcome of this study was QoL, as measured by the IBS-36 [19] and the SF-36 [20]. The IBS-36 has high test-retest reliability, internal consistency, content validity, convergent and divergent construct validities, sensitivity to changes in symptom severity, and high correlation with the SF-36 [19]. This test also queries multiple symptoms for persons suffering from GI disorders and is highly correlated with patient-reported symptoms [19]. The IBS-36 provides one total score (possible range of 0 - 216) with a lower score indicating a better QoL. The SF-36 provides psychometrically-based physical and mental health summary measures and a preference-based health utility index. The SF-36 includes a $\mathrm{t}$-score for each scale or domain ranging from $0-100$ with higher scores representing better perceived QoL. The SF-36 is a general QoL measure that is commonly used, but it does not address areas of concern for patients with GI disorders, like food avoidance and bowel-specific problems [19]. It also does not target a specific age, disease, or treatment group [20].

Subjects were instructed on how to complete a 3-day food record (two weekdays and one weekend day to allow for fluctuations over a normal weekly period) at each assessment in order to list all food and beverage consumption during that particular time. The 3-day food record was used at each assessment to gauge compliance to the elimination diet based on a comparison of the foods eaten during those three days to the ImmunoBloodprint results of reactive foods for that subject. For example, if a subject ate 20 different foods during the 3-day period and one of the foods was IgG-reactive according to the ImmunoBloodprint results, then the subject was 95\% compliant with the diet for that particular assessment. Subjects were assessed at baseline and 30-, 60-, and 90-day follow-up.

\subsection{Statistical Analysis}

Data were analyzed using SPSS 19 (IBM Inc., Chicago, IL) for Windows. Frequency and descriptive statistics were calculated on all variables. We utilized linear mixed modeling (LMM) to assess the fixed effect of time on changes in our outcome variables from baseline to the 90-day follow-up period. If the type III test of the fixed effect of time and the parameter estimate of the baseline to the 90-day fixed effect were significant, then we used pairwise comparisons to determine the unique differences between baseline and follow-up values at 30,60, and 90 days. LMM allowed us to account for subject attrition, inter-correlated responses between time points, and non-constant variability. The criterion for statistical significance was $\alpha=0.05$.

\section{RESULTS}

\subsection{Prevalence of IgG-Reactive Foods}

For all participants, the average number of IgG-reactive foods and ingredients was $14.3(\mathrm{SD}=6.2, \mathrm{R}=5,31)$. Average percent compliance to the diet was as follows: 30-day follow-up, $95.8 \%(\mathrm{SD}=6.6, \mathrm{R}=76.92,100)$; 60-day follow-up, $95.8 \%(\mathrm{SD}=7.4, \mathrm{R}=71.4,100)$; and 90-day follow-up, 95.5\% $(\mathrm{SD}=5.2, \mathrm{R}=85.7,100)$. Figure 1 shows the top 10 most frequently tested IgGreactive foods and ingredients for all participants.

\subsection{Quality of Life}

Figure 2 shows the IBS-36 total score at baseline and 30-, 60-, and 90-day follow-up. For the IBS-36 total score, a significant fixed effect was found for time (F[3, $7.3]=24.3, p<0.001)$, and the parameter estimate between baseline and 90-day follow-up was also significant $(\mathrm{t}[32.8]=8.5, \mathrm{p}<0.001)$. Pairwise comparisons revealed that the IBS-36 total score at baseline was significantly higher than at 30 days (mean difference $=26.0 ; \mathrm{SE}=4.6$; $95 \%$ CI: $-12.8,39.0 ; \mathrm{p}<0.001$ ), at 60 days (mean difference $=30.8 ; \mathrm{SE}=3.8 ; 95 \%$ CI: 20.1, 41.5; $\mathrm{p}<0.001)$, and at 90 days (mean difference $=44.3 ; \mathrm{SE}=5.2 ; 95 \%$ CI: $-29.7,58.9 .0 ; \mathrm{p}<0.001)$.

Figure 3 shows the descriptive values of all eight scales on the SF-36 at baseline and 30-, 60-, and 90-day follow-up. For physical functioning, a significant fixed effect was found for time $(F[3,15.8]=4.9, \mathrm{p}=0.01)$,



Figure 1. The top $10 \mathrm{IgG}$-reactive foods and ingredients among participants. 


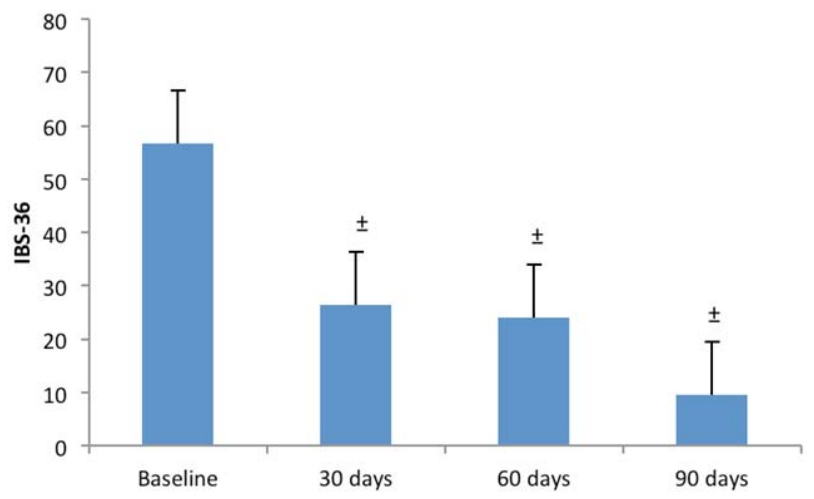

Figure 2. IBS-36 at baseline and 30, 60, and 90 days. Results of linear mixed modeling; \pm significantly different from baseline, $\mathrm{p}<0.001$.

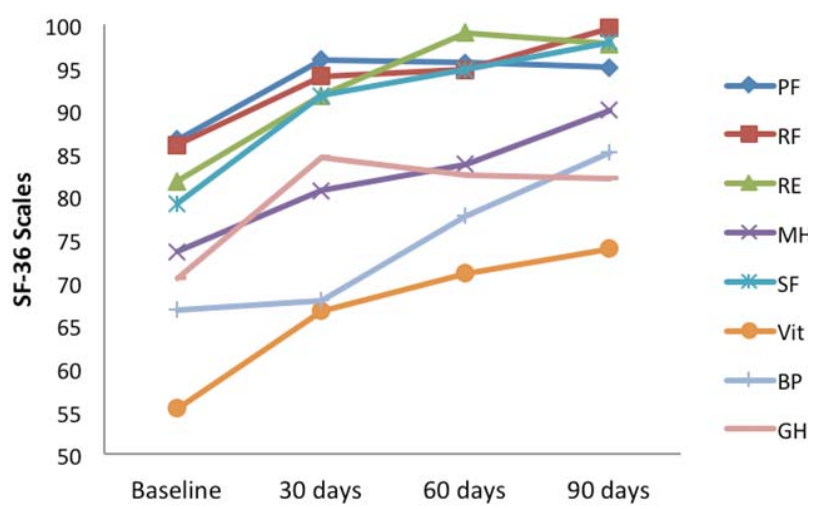

Figure 3. SF-36 scales at baseline and 30, 60 and 90 days. Results of linear mixed modeling; PF-Physical Functioning; RF-Role Physical; RE-Role Emotional; MH-Mental Health; SF-Social Functioning; Vit-Vitality; BP-Bodily Pain; GH-General Health.

and the parameter estimate between baseline and 90-day follow-up was also significant $(\mathrm{t}[32.3]=-3.6, \mathrm{p}=0.001)$. Pairwise comparisons revealed that physical functioning at baseline was significantly lower than at 30 days (mean difference $=-6.2 ; \mathrm{SE}=1.8 ; 95 \% \mathrm{CI}:-11.45,-1.0 ; \mathrm{p}=$ 0.01 ), at 60 days (mean difference $=-6.2 ; \mathrm{SE}=1.6 ; 95 \%$ CI: $-10.8,-1.6 ; \mathrm{p}<0.05$ ), and at 90 days (mean difference $=-8.0 ; \mathrm{SE}=2.15 ; 95 \% \mathrm{CI}:-14.0,-1.9 ; \mathrm{p}<0.01)$. For role-physical, a significant fixed effect was found for time $(F[3,26.4]=9.7, p<0.001)$, and the parameter estimate between baseline and 90-day follow-up was also significant $(\mathrm{t}[72.1]=-5.0, \mathrm{p}<0.001)$. Pairwise comparisons revealed that role-physical at baseline was significantly lower than at 90 days only (mean difference $=$ -14.4 ; $\mathrm{SE}=2.9$; 95\% CI: $-22.2,-6.5 ; \mathrm{p}<0.001)$. For role-emotional, a significant fixed effect was found for time $(F[3,21.8]=14.9, p<0.001)$, and the parameter estimate between baseline and 90-day follow-up was also significant $(\mathrm{t}[50.4]=-6.0, \mathrm{p}<0.001)$. Pairwise comparisons revealed that role-emotional at baseline was significantly lower at 30 days (mean difference $=-8.2$; $\mathrm{SE}$
$=2.2 ; 95 \% \mathrm{CI}:-14.0,-2.4 ; \mathrm{p}=0.001)$, at 60 days (mean difference $=-16.5 ; \mathrm{SE}=2.6 ; 95 \% \mathrm{CI}:-23.6,-9.5 ; \mathrm{p}<$ 0.001 ), and at 90 days (mean difference $=-15.7 ; \mathrm{SE}=$ 2.6; 95\% CI: $-22.9,-8.6 ; \mathrm{p}<0.001)$. For mental health, a significant fixed effect was found for time $(F[3,27.0]=$ $32.5, \mathrm{p}<0.001$ ), and the parameter estimate between baseline and 90-day follow-up was also significant $\mathrm{t}[$ [65.0] $=-7.75, \mathrm{p}<0.001)$. Pairwise comparisons revealed that mental health at baseline was significantly lower at 60 days (mean difference $=-7.8 ; \mathrm{SE}=2.5 ; 95 \% \mathrm{CI}:-14.9$, $-0.7 ; \mathrm{p}<0.05$ ) and at 90 days (mean difference $=-15.4$; $\mathrm{SE}=2.0 ; 95 \% \mathrm{CI}:-20.8,-10.0 ; \mathrm{p}<0.001)$. For social functioning, a significant fixed effect was found for time $(F[3,24.6]=22.6, p<0.001)$, and the parameter estimate between baseline and 90-day follow-up was also significant $(\mathrm{t}[60.1]=-7.7, \mathrm{p}<0.001)$. Pairwise comparisons revealed that social functioning at baseline was significantly lower than at 30 days (mean difference $=-12.7$; $\mathrm{SE}=2.8 ; 95 \% \mathrm{CI}:-20.5,-4.9 ; \mathrm{p}<0.001)$, at 60 days (mean difference $=-19.7 ; \mathrm{SE}=4.3 ; 95 \% \mathrm{CI}:-32.1,-7.3$; $\mathrm{p}=0.001$ ), and at 90 days (mean difference $=-18.3$; SE $=2.3 ; 95 \%$ CI: $-24.7,-11.8 ; \mathrm{p}<0.001)$. For vitality, a significant fixed effect was found for time $(F[3,23.9]=$ $9.2, \mathrm{p}<0.001$ ), and the parameter estimate between baseline and 90-day follow-up was also significant $(\mathrm{t}[31.5]=$ $-5.1, \mathrm{p}<0.001)$. Pairwise comparisons revealed that vitality at baseline was slightly lower at 30 days (mean difference $=-9.7 ; \mathrm{SE}=3.7 ; 95 \% \mathrm{CI}:-20.3,0.81 ; \mathrm{p}=$ 0.09 ), but significantly lower at 60 days (mean difference $=-14.0 ; \mathrm{SE}=3.1 ; 95 \% \mathrm{CI}:-22.7,-5.3 ; \mathrm{p}<0.001)$ and at 90 days (mean difference $=-18.7 ; \mathrm{SE}=3.6 ; 95 \% \mathrm{CI}$ : $-28.8,-8.7 ; \mathrm{p}<0.001)$. For bodily pain, a significant fixed effect was found for time $(F[3,28.6]=23.6, p<$ 0.001 ), and the parameter estimate between baseline and 90-day follow-up was also significant $(\mathrm{t}[59.4]=-7.0, \mathrm{p}$ $<0.001$ ). Pairwise comparisons revealed that bodily pain at baseline was significantly lower at 60 days (mean difference $=-10.5 ; \mathrm{SE}=3.3 ; 95 \% \mathrm{CI}:-19.8,-1.2 ; \mathrm{p}<0.05)$ and at 90 days (mean difference $=-17.8 ; \mathrm{SE}=2.5 ; 95 \%$ CI: $-24.7,-10.9 ; \mathrm{p}<0.001)$. For general health, a significant fixed effect was found for time $(\mathrm{F}[3,19.9]=7.0$, $\mathrm{p}<0.01$ ), and the parameter estimate between baseline and 90-day follow-up was also significant $\mathrm{t}[\mathrm{t} 22.7]=-2.9$, $\mathrm{p}=0.01$ ). Pairwise comparisons revealed that general health at baseline was significantly lower than at 30 days (mean difference $=-6.3 ; \mathrm{SE}=1.4 ; 95 \% \mathrm{CI}:-10.5,-2.1$; $\mathrm{p}=0.001$ ) and at 60 days (mean difference $=-5.6 ; \mathrm{SE}=$ 1.8; $95 \%$ CI: $-10.9,-0.27 ; \mathrm{p}<0.05)$ and slightly nonsignificant at 90 days (mean difference $=-5.9 ; \mathrm{SE}=2.1$; 95\% CI: $-12.0,0.1 ; \mathrm{p}=0.06$ ).

\section{DISCUSSION}

In this clinic-based study of persons with unresolved GI disorders, we assessed the effect of an IgG-mediated 
food sensitivity test, the ImmunoBloodprint, combined with a subsequent elimination diet for 90 days on QoL. Eliminating foods to which participants were sensitive, as identified by the IgG-mediated ImmunoBloodprint test, resulted in clinically-significant improvement in QoL, as measured by the IBS-36 and the SF-36. The changes found on the IBS-36 and SF-36 in our study suggest that not only were our participants making improvements, but they were subjectively associating those changes with how they felt in multiple domains. Additionally, the use of self-report QoL instruments to assess GI disorders is increasing, given that the patient's perspective is critical in any health condition [21]. The reported increase in QoL may equate to increased productivity, less absentteeism from work, less utilization of medical specialists, and an overall reduction on the economic burden of this disease.

Although IgE testing is the most commonly recognized method to determine food hypersensitivity [22,23], the use of $\operatorname{IgG}$ testing may also be useful in identifying foods that are reactive [21,24]. Despite their potential usefulness as a clinical tool, the medical community does not universally accept the use of IgG-mediated testing since IgG has been found in healthy individuals after ingestion of foods [25-27]. In addition, it is unknown what percentage of the population with high levels of IgG food-related antibodies has unhealthy bowel symptoms. Furthermore, inter-laboratory reproducibility is lacking, and the sensitivity and specificity of IgG food hypersensitivity testing need to be evaluated [21].

Nonetheless, this study demonstrates that eliminating foods that are IgG-reactive, while replacing them with similar, non-reactive foods to prevent nutrient deficiencies, can be a novel strategy for improving QoL in patients with unresolved GI disorders. It is possible that QoL is improved by reducing symptomatology like abnormal colonic motility manifested by more frequent and stronger bowel contractions and shorter colonic transit time that may be explained by IgG sensitivity exhibited to certain foods. This method is a relatively low-cost strategy that may be useful when combined with other existing approaches to the illness. Food exclusion diets require strict adherence, changing eating patterns, selfdiscipline, will-power, and motivation, but the reported high compliance to the diet ( $>95 \%$ average compliance) in our study demonstrates their feasibility. Our high compliance rate may have been due to test results guiding the food elimination diet [28], instead of trial and error, and/ or immediate positive response as seen in self-reported QoL. Therefore, IgE and IgG food testing can help direct the food exclusion diet initially; however, the only modality for truly identifying a clinical reaction to foods is through oral food challenge [29,30].

Food challenge follows an elimination diet by adding back to the diet the potentially reactive foods. Once the offending foods have been identified, they may be restored to the diet on a rotation schedule of $3-4$ days between ingestion. This rotation method is utilized to prevent GI symptom reoccurrence, which occurs by possible accumulation of antibody-antigen complexes due to reactive foods eaten on successive days [21]. Even though our study did not employ food challenge after the elimination diet, as other studies have $[21,24]$, our results provide evidence that the ImmunoBloodprint could be effective in guiding exclusion diets for this purpose. Studies employing blinded food challenges are also necessary to corroborate IgG testing results and thus aid in overcomeing the bias against this potentially useful tool. Hence, further studies are warranted in which the ImmunoBloodprint test is used to guide exclusion diets and food challenges.

\section{LIMITATIONS}

Limitations of this study include our lack of any additional biological markers of inflammation, e.g., C-reactive protein, cytokines, or growth factors, to be able to determine the possible relationship between changes in QoL with indicators of chronic inflammation. For example, the decreased release of inflammatory mediators, like cytokines and lymphocyte proliferation responses, can be measured after food elimination diets [31,32]. Some studies have suggested the need to evaluate histamine, substance $\mathrm{P}$, and serotonin production as other key biological indicators [21]. Another limitation of this study is the lack of a control group of participants with unresolved GI disorders on a "sham" diet. Previous food elimination diets have reported that patients on sham diets also improved to a lesser extent in QoL measures, highlighting the importance of performing double-blind, randomized controlled trials in order to prevent overestimation of the potential benefit of the intervention [21]. Participants were not re-assessed with another IgG test at 90 days to determine consistency across the study and/or to denote any changes in response to the intervention. Lastly, we included participants who self-reported GI disorders and were not diagnosed by ROME criteria for functional GI disorders. However, most patients with GI disorders, including IBS, are undiagnosed [9], making it difficult to entirely delineate the population characteristics. While this may call into question the direct clinical relevance of our findings to a more standardized population, our study sample included twice as many females as males, which is similar to the IBS population.

\section{CONCLUSION}

The results of our study suggest that utilizing an elimination diet of IgG-reactive foods, detected with the Im- 
munoBloodprint test, in participants with unresolved self-reported GI disorders improves QoL measures. Thus, this trial demonstrated that IgG-mediated testing combined with a subsequent elimination diet may offer the opportunity for such patients to improve their dietary behaviors and symptoms by utilizing a tailored, individually-specific program. This dietary strategy, offering considerable benefit to patients with GI disorders as shown in this and previous studies [28], can represent a tool worthy of further clinical and biomedical research. Future research should focus on randomized controlled trials using double-blind methods and patients identified with ROME criteria. Additional studies are necessary to explore the correlation between IgG food sensitivity and abnormalities in colonic motility. Finally, future analyses may determine if improved patient QoL scores translate into reduced cost of IBS to the patient, employer, and the overall healthcare system.

\section{ACKNOWLEDGEMENTS}

We are thankful to all the volunteers who participated in this study. This study was supported by a gift from Immuno Laboratories of Ft. Lauderdale, FL.

\section{REFERENCES}

[1] Owens, D.M., Nelson, D.K. and Talley, N.J. (1995) The irritable bowel syndrome: Long-term prognosis and the physician-patient interaction. Annals of Internal Medicine, 122, 107-112. doi:10.7326/0003-4819-122-2-199501150-00005

[2] Hahn, B.A., Yan, S. and Strassels, S. (1999) Impact of irritable bowel syndrome on quality of life and resource use in the United States and United Kingdom. Digestion, 60, 77-81. doi:10.1159/000007593

[3] El-Serag, H.B., Olden, K. and Bjorkman, D. (2002) Health-related quality of life among persons with irritable bowel syndrome: A systematic review. Alimentary Pharmacology \& Therapeutics, 16, 1171-1185. doi:10.1046/j.1365-2036.2002.01290.x

[4] Camilleri, M., Northcutt, A.R., Kong, S., Dukes, G.E., McSorley, D. and Mangel, A.W. (2000) Efficacy and safety of alosetron in women with irritable bowel syndrome: A randomised, placebo-controlled trial. Lancet, 355, 1035-1040. doi:10.1016/S0140-6736(00)02033-X

[5] Watson, M.E., Lacey, L., Kong, S., Northcutt, A.R., McSorley, D., Hahn, B. and Mangel, A.W. (2001) Alosetron improves quality of life in women with diarrheapredominant irritable bowel syndrome. The American Journal of Gastroenterology, 96, 455-459. doi:10.1111/j.1572-0241.2001.03525.x

[6] Van, O.M., Mayeur, S., Meeus, M.A., Rosillon, D., Hendrickx, B. and Ceuppens, M. (1995) A double-blind crossover comparison study of the safety and efficacy of mebeverine with mebeverine sustained release in the treatment of irritable bowel syndrome. Journal of Clini- cal Pharmacy and Therapeutics, 20, 277-282. doi:10.1111/j.1365-2710.1995.tb00663.x

[7] Inauen, W. and Halter, F. (1994) Clinical efficacy, safety and tolerance of mebeverine slow release $(200 \mathrm{mg}) \mathrm{vs}$. mebeverine tablets in patients with irritable bowel syndrome. Drug Investigation, 8, 234-240. doi:10.1007/BF03258483

[8] Gilbody, J.S., Fletcher, C.P., Hughes, I.W. and Kidman, S.P. (2000) Comparison of two different formulations of mebeverine hydrochloride in irritable bowel syndrome. International Journal of Clinical Practice, 54, 461-464.

[9] Lembo, A.J. (2007) The clinical and economic burden of irritable bowel syndrome. Practical Gastroenterology, 9, 3-9.

[10] Cremonini, F. and Talley, N.J. (2005) Irritable bowel syndrome: Epidemiology, natural history, health care seeking and emerging risk factors. Gastroenterology Clinics of North America, 34, 189-204. doi:10.1016/j.gtc.2005.02.008

[11] Talley, N.J. (1999) Irritable bowel syndrome: Definition, diagnosis and epidemiology. Best Practice \& Research Clinical Gastroenterology, 13, 371-384. doi:10.1053/bega.1999.0033

[12] Petitpierre, M., Gumowski, P. and Girard, J.P. (1985) Irritable bowel syndrome and hypersensitivity to food. Annals of Allergy, 54, 538-540.

[13] Zar, S., Kumar, D. and Benson, M.J. (2001) Food hypersensitivity and irritable bowel syndrome. Alimentary Pharmacology \& Therapeutics, 15, 439-449. doi:10.1046/j.1365-2036.2001.00951.x

[14] Zwetchkenbaum, J.F. and Burakoff, R. (1988) Food allergy and the irritable bowel syndrome. The American Journal of Gastroenterology, 83, 901-904.

[15] Awazuhara, H., Kawai, H. and Maruchi, N. (1997) Major allergens in soybean and clinical significance of IgG4 antibodies investigated by IgE- and IgG4-immunoblotting with sera from soybean-sensitive patients. Clinical \& Experimental Allergy, 27, 325-332. doi:10.1111/j.1365-2222.1997.tb00711.x

[16] Crowe, S.E. and Perdue, M.H. (1992) Gastrointestinal food hypersensitivity: Basic mechanisms of pathophysiology. Gastroenterology, 103, 1075-1095.

[17] elRafei, A., Peters, S.M., Harris, N. and Bellanti, J.A. (1989) Diagnostic value of IgG4 measurements in patients with food allergy. Annals of Allergy, 62, 94-99.

[18] Host, A., Husby, S., Gjesing, B., Larsen, J.N. and Lowenstein, H. (1992) Prospective estimation of IgG, IgG subclass and $\operatorname{IgE}$ antibodies to dietary proteins in infants with cow milk allergy. Levels of antibodies to whole milk protein, BLG and ovalbumin in relation to repeated milk challenge and clinical course of cow milk allergy. Allergy, 47, 218-229. doi:10.1111/j.1398-9995.1992.tb00654.x

[19] Groll, D., Vanner, S.J., Depew, W.T., DaCosta, L.R., Simon, J.B., Groll, A., Roblin, N. and Paterson, W.G. (2002) The IBS-36: A new quality of life measure for irritable bowel syndrome. The American Journal of Gastroenterology, 97, 962-971. doi:10.1111/j.1572-0241.2002.05616.x 
[20] Ware, J., Kosinski, M. and Dewey, J. (2000) How to score version two of the sf-36 health survey. Quality Metric, Incorporated, Lincoln, RI.

[21] Drisko, J., Bischoff, B., Hall, M. and McCallum, R. (2006) Treating irritable bowel syndrome with a food elimination diet followed by food challenge and probiotics. Journal of the American College of Nutrition, 25, 514-522. doi:10.1080/07315724.2006.10719567

[22] Asero, R., Ballmer-Weber, B.K., Beyer, K., Conti, A., Dubakiene, R., Fernandez-Rivas, M., Hoffmann-Sommergruber, K., Lidholm, J., Mustakov, T., Oude Elberink, J.N., Pumphrey, R.S., Stahl, S.P., van, R.R., Vlieg-Boerstra, B.J., Hiller, R., Hourihane, J.O., Kowalski, M., Papadopoulos, N.G., Wal, J.M., Mills, E.N. and Vieths, S. (2007) IgE-mediated food allergy diagnosis: Current status and new perspectives. Molecular Nutrition \& Food Research, 51, 135-147. doi:10.1002/mnfr.200600132

[23] Ortolani, C., Bruijnzeel-Koomen, C., Bengtsson, U., Bindslev-Jensen, C., Bjorksten, B., Host, A., Ispano, M., Jarish, R., Madsen, C., Nekam, K., Paganelli, R., Poulsen, L.K. and Wuthrich, B. (1999) Controversial aspects of adverse reactions to food. European Academy of Allergology and Clinical Immunology (EAACI) reactions to food subcommittee. Allergy, 54, 27-45. doi:10.1034/j.1398-9995.1999.00913.x

[24] Atkinson, W., Sheldon, T.A., Shaath, N. and Whorwell, P.J. (2004) Food elimination based on IgG antibodies in irritable bowel syndrome: A randomised controlled trial. Gut, 53, 1459-1464. doi:10.1136/gut.2003.037697

[25] Husby, S., Oxelius, V.A., Teisner, B., Jensenius, J.C. and
Svehag, S.E. (1985) Humoral immunity to dietary antigens in healthy adults. Occurrence, isotype and IgG subclass distribution of serum antibodies to protein anti- gens. International Archives of Allergy and Immunology, 77, 416-422. doi:10.1159/000233819

[26] King, H.C. (1994) Exploring the maze of adverse reactions to foods. Ear, Nose \& Throat Journal, 73, 237-241.

[27] Kruszewski, J., Raczka, A., Klos, M. and Wiktor-Jedrzejczak, W. (1994) High serum levels of allergen specific IgG-4 (asIgG-4) for common food allergens in healthy blood donors. Archivum Immunologiae et Therapia Experimentalis (Warsz), 42, 259-261.

[28] Nanda, R., James, R., Smith, H., Dudley, C.R. and Jewell, D.P. (1989) Food intolerance and the irritable bowel syndrome. Gut, 30, 1099-1104. doi:10.1136/gut.30.8.1099

[29] Sicherer, S.H. (2001) Clinical implications of cross-reactive food allergens. Journal of Allergy and Clinical Immunology, 108, 881-890. doi:10.1067/mai.2001.118515

[30] Sicherer, S.H. (1999) Food allergy: When and how to perform oral food challenges. Pediatric Allergy and Immunology, 10, 226-234. doi:10.1034/j.1399-3038.1999.00040.x

[31] Gordon, B.R. (2003) Approaches to testing for food and chemical sensitivities. Otolaryngologic Clinics of North America, 36, 917-940. doi:10.1016/S0030-6665(03)00059-8

[32] Sampson, H.A. and Burks, A.W. (1996) Mechanisms of food allergy. Annual Review of Nutrition, 16, 161-177. doi:10.1146/annurev.nu.16.070196.001113 


\section{ABBREVIATIONS}

Gastrointestinal (GI)

Irritable bowel syndrome (IBS)

Quality of life (QoL)

Immunoglobulin E (IgE)

Immunoglobulin $\mathrm{G}$ (IgG)

Irritable Bowel Syndrome-36 (IBS-36)
Short-Form Health Survey 36 (SF-36)

Standard deviation (SD)

Range (R)

Statistical package for social sciences (SPSS)

Linear mixed modeling (LMM)

Confidence interval (CI)

Standard error (SE)

APPENDIX 1. The 115 tested foods comprising the ImmunoBloodprint.

\begin{tabular}{|c|c|c|c|c|}
\hline Alfalfa & Celery & Herring & Parsley & Seed, Rape (Canola) \\
\hline Almond & Cheese & Lamb & Pea & Sesame \\
\hline Amaranth & Cherry & Lemon & Peach & Shrimp \\
\hline Apple & Chicken & Lentil & Peanut & Snapper \\
\hline Asparagus & Cinnamon & Lettuce & Pecan & Sole \\
\hline Avocado & Clam & Lime & Pepper, B/W & Soybean \\
\hline Banana & Clove & Lobster & Pepper, Chili & Spinach \\
\hline Barley & Cocoa & Mackerel & Pepper, Green & Strawberry \\
\hline Bean, Green & Chocolate & Milk, Cow’s & Perch & Sugar, Cane \\
\hline Bean, Kidney & Coconut & Milk, Goat's & Pineapple & Sunflower \\
\hline Bean, Lima & Cod & Millet & Plum & Tangerine \\
\hline Bean, Mung & Coffee & Mushroom & Pork & Tea \\
\hline Bean, Pinto & Corn & Mustard & Potato, Sweet & Tomato \\
\hline Bean, Yellow Wax & Crab & Nut, Brazil & Potato, White & Trout \\
\hline Beef & Cranberry & Nut, Cashew & Pumpkin & Tuna \\
\hline Beet & Egg & Nutmeg & Quinoa & Turkey \\
\hline Broccoli & Eggplant & Oat & Radish & Walnut \\
\hline Brussels Sprouts & Flounder & Olive & Rice & Wheat \\
\hline Buckwheat & Garlic & Onion & Rye & White Fish \\
\hline Cabbage & Ginger & Orange & Safflower & Yam \\
\hline Cantaloupe & Grape & Oregano & Sage & Yeast, Baker's \\
\hline Carrot & Grapefruit & Oyster & Salmon & Yeast, Brewer's \\
\hline Cauliflower & $\begin{array}{c}\text { Haddock } \\
\text { Halibut }\end{array}$ & Papaya & Scallops & Zucchini \\
\hline
\end{tabular}

